Case Report

\title{
Gastric Outlet Obstruction Caused by a Triple Lesion: Eosinophilic Gastroenteritis, Brunner's Gland Hyperplasia and Pancreatic Heterotopia in Unison
}

\author{
Apurva Sharma ${ }^{1, ~ *, ~ S o n m o o n ~ M o h a p a t r a ~}{ }^{1}$, Kheng-Jim Lim², Abhijeet Chaubal ${ }^{3}$, Arkady Broder $^{4}$ \\ ${ }^{1}$ Department of Medicine, Saint Peters University Hospital, New Brunswick, USA \\ ${ }^{2}$ Department of Medicine, Division Gastroenterology and Hepatology, Rutgers Medical School Robert Wood Johnson, New Brunswick, USA \\ ${ }^{3}$ Department of Pathology, Saint Peters University Hospital, New Brunswick, USA \\ ${ }^{4}$ Department of Medicine, Division of Gastroenterology and Hepatology, Saint Peters University Hospital, New Brunswick, USA
}

\section{Email address:}

apurva.doc@gmail.com (A. Sharma)

${ }^{*}$ Corresponding author

\section{To cite this article:}

Apurva Sharma, Sonmoon Mohapatra, Kheng-Jim Lim, Abhijeet Chaubal, Arkady Broder. Gastric Outlet Obstruction Caused by a Triple Lesion: Eosinophilic Gastroenteritis, Brunner's Gland Hyperplasia and Pancreatic Heterotopia in Unison. Journal of Surgery. Vol. 5, No. 3, 2017, pp. 43-46. doi: 10.11648/j.js.20170503.12

Received: March 1, 2017; Accepted: March 30, 2017; Published: May 3, 2017

\begin{abstract}
Gastric outlet obstruction is a common entity described with numerous benign and malignant causes. Historically, benign causes include Peptic Ulcer disease (PUD); however recent cases have reported other causes including Eosinophilic Gastroenteritis (EGE), Brunner's Gland Hyperplasia (BGH) and Pancreatic Heterotopia (PH). We present an unusual case of a 31 year old male patient with severe gastric outlet obstruction requiring surgical intervention caused by all three pathologies in unison - EGE, BGH and PH. The patient underwent surgical resection with distal gastrectomy and Billroth II reconstruction. Histology confirmed the three pathologies and post-operative recovery of the patient was uneventful.
\end{abstract}

Keywords: Eosinophilic Gastroenteritis, Brunner’s Gland Hyperplasia, Pancreatic Heterotopia

\section{Introduction}

Gastric Outlet Obstruction (GOO) is a clinical and pathophysiological syndrome due to a mechanical obstruction. There are several causes listed for GOO including benign as well as malignant lesions. Prior to the advent of Proton Pump Inhibitor (PPI), benign causes were seen in most of the cases with Peptic Ulcer Disease (PUD) as the most common. However recently, majority of cases are attributable to malignancy including pancreatic adenocarcinoma and gastric cancer. Rare benign causes are Eosinophilic Gastroenteritis (EGE), Brunner's Gland Hyperplasia (BGH) and Pancreatic Heterotopia (PH). EGE is a rare disease that has been reported as a cause of GOO [1]. BGH is a benign lesion that is mostly asymptomatic. However, occasionally they can attain larger sizes and cause symptoms of obstruction $[5,6]$. $\mathrm{PH}$ is a rare condition which is generally asymptomatic, but may present as a cause of GOO $[8,9]$. We present an unusual case where all the three rare entities caused pyloric obstruction in unison.

\section{Case Report}

A 31 year old Indian gentleman presented to the emergency department with complaint of vomiting for 20 days. He described 10-15 episodes of postprandial vomiting per day associated with dull upper abdominal pain which poorly responded to any medication. He had similar presentation 2 weeks prior for which he received only symptomatic treatment He did not complain of any weight loss and diarrhea. Social history was unremarkable. On physical examination, the patient was afebrile with normal vital signs. The abdomen was non-distended but tender in the epigastric region. Routine blood tests were indicative of severe dehydration and hypokalemia with metabolic alkalosis. Hepatic panel was 
normal. A right upper quadrant ultrasound showed no evidence of cholelithiasis and abdominal CT was normal. An esophagogastroduodenoscopy showed LA grade D esophagitis with a mass in the gastric antrum causing difficulty to pass the scope beyond the mass (Figure 1). The biopsy showed foveolar cell hyperplasia consistent with hyperplastic polyp and absence of helicobacter pylori infection. His symptoms persisted despite conservative

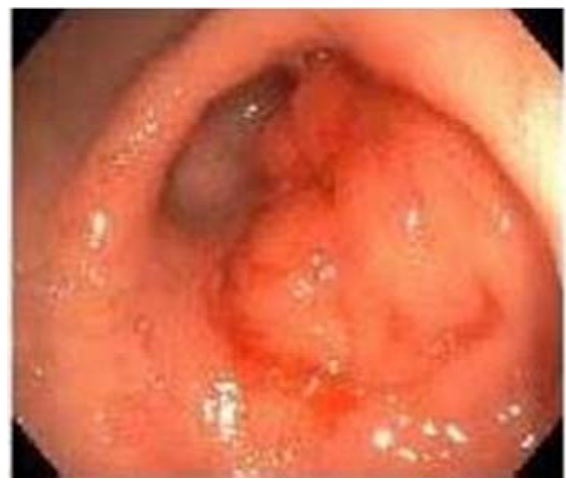

measures. Hence, he underwent surgical resection with distal gastrectomy and billroth II reconstruction. The resected specimen consisted of distal part of stomach with pylorus and proximal duodenal cuff. Histology was suggestive of marked eosinophilic infiltrates consistent with EGE (Figure 2). The duodenal tissue also showed BGH and PH (Figure 3, 4). Post operative period was uncomplicated with complete resolution of symptoms.

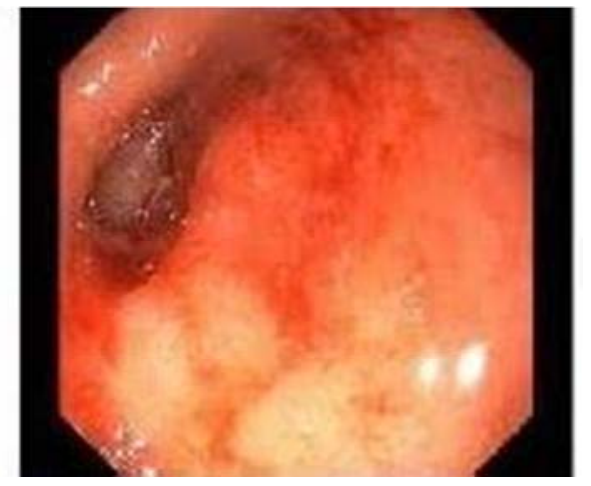

Figure 1. Endoscopic image of inflammatory mass in the gastric antrum with severe stenosis. [Pre and post pinch biopsy].

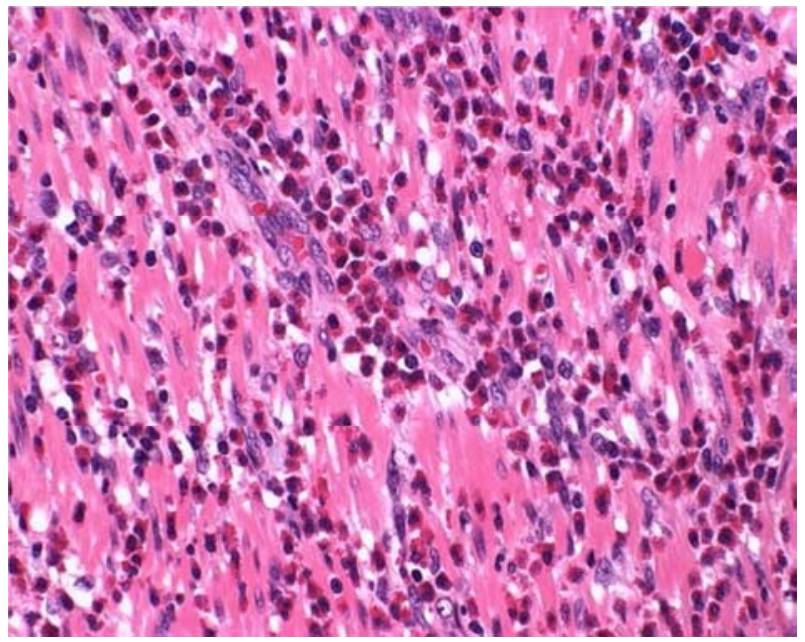

Figure 2. High power view showing Eosinophilic infiltrates within the muscularis propria.

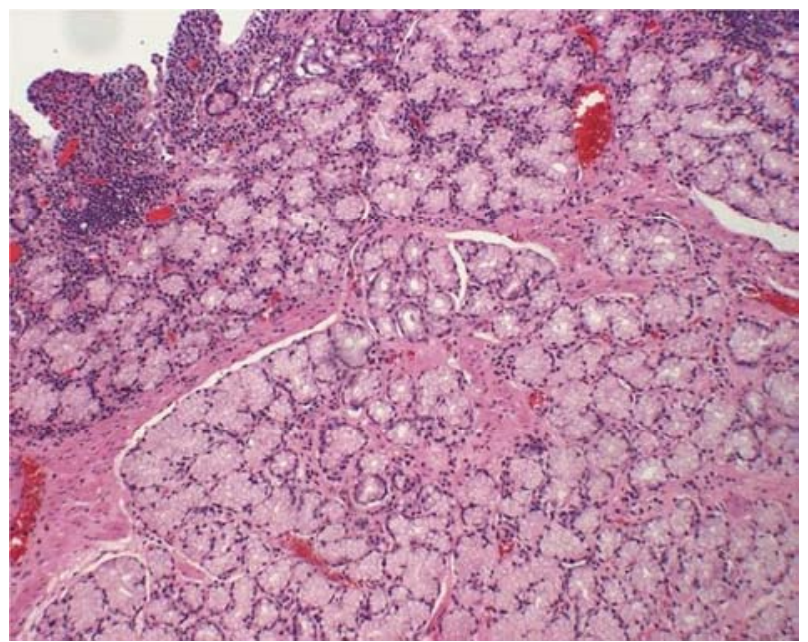

Figure 3. Low power view of the mucosa showing BGH and the crypts with Eosinophilic infiltrates.

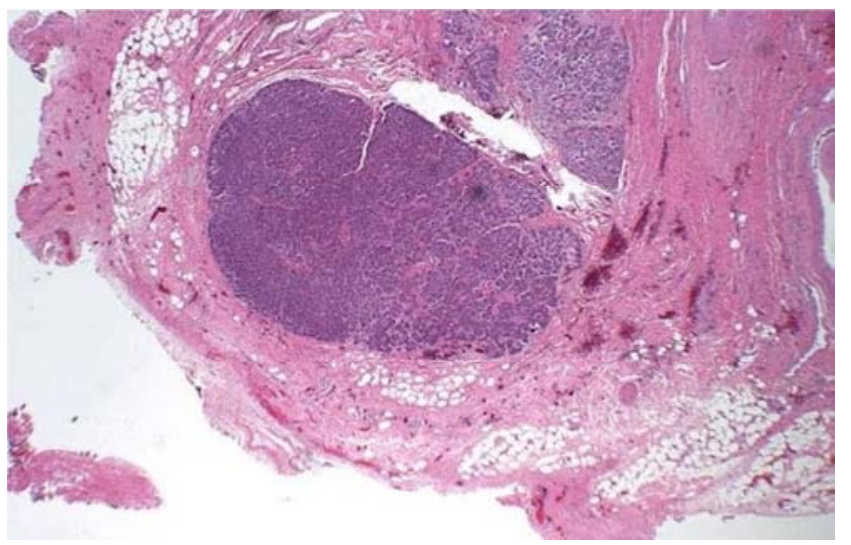

Figure 4. Low power view demonstrating duodenal tissue with pancreatic heterotopia.

\section{Discussion}

GOO is the clinical and pathological sequence of any disease process that produces a mechanical impediment to gastric emptying [1]. Clinical entities that can cause GOO are divided into two well defined groups- benign and malignant. Malignancy remains the most common cause in adults; however, a significant number of patients with benign diseases present with GOO. The most common benign causes are PUD and ingestion of caustics. Other causes include gastric polyps, pyloric stenosis, congenital duodenal webs, gallstone obstruction (Bouveret syndrome), pancreatic pseudocysts, and bezoars. Rare pathologies as EGE, BGH and $\mathrm{PH}$ have independently manifested as a cause of GOO.

EGE seldom manifests as GOO [2]. EGE is a rare entity characterized by eosinophilic infiltration of areas of the GI tract in absence of parasitic or extra-intestinal disease. The presentation of EGE varies according to the anatomic location of eosinophilic infiltration in different layers of intestinal wall 
- the mucosa, muscularis and subserosal layer. The muscularis layer is involved in approximately $10-60 \%$ of cases and usually associated with obstructive symptoms secondary to pyloric stenosis, gastric outlet obstruction and rarely, intussusception [3]. The etiology and pathogenesis are not well understood and there is an association with hypersensitivity reactions leading to excessive accumulation of eosinophils. Diagnosis is generally based on high clinical suspicion, associated laboratory, endoscopic and biopsy findings. Laparoscopic full-thickness biopsies may be necessary to diagnose muscularis and subserosal forms of EGE [3]. Endoscopy usually shows erythematous, friable, sometimes nodular mucosa and rarely ulceration in the stomach. The most common histologic findings are crypt hyperplasia and eosinophilic infiltration in the lamina propria. Occasionally, a large ulcerative mass with obstruction can be seen. Microscopy usually shows an eosinophil count of 20 or more per high-power field [3]. Treatment is often based on the severity of symptoms and is managed with avoidance of inciting food allergens. Though corticosteroids are the mainstay of therapy in patients with moderate to severe disease, various types of gastrectomies including Billroth II reconstruction have been done in patients with undiagnosed EGE [3]. Similarly, in our case, the endoscopic biopsies were negative as there was no mucosal infiltration. Therefore, corticosteroid was never administered as the clinical scenario and endoscopic biopsy result did not support the diagnosis of EGE. However, the histology of the resected specimen of distal stomach was consistent with EGE suggesting the limitation of endoscopic biopsy in the diagnosis of EGE specifically in mucosal and muscularis disease [4].

$\mathrm{BGH}$ is a benign lesion generally arising from the duodenum. The Brunner's gland is found in the proximal duodenum and protects the duodenal lining from gastric acid by secreting alkaline-based mucus. It accounts for approximately $10.6 \%$ of benign tumors in the duodenum [5]. The pathogenesis of Brunner's gland hyperplasia is not well known. However, it is hypothesized that excess gastric acid secretion or increased inflammation may lead to hyperplasia [5]. Most of the lesions are less than $1 \mathrm{~cm}$ in size and asymptomatic. Occasionally, it causes symptoms of obstruction and abdominal pain [6, 7]. Endoscopy has a sensitivity of $72-89 \%$, however biopsies may be non-diagnostic as lesions are submucosal and may be missed on pinch biopsy which is one of the possibilities occurred in our case. The obstruction was so severe that the scope didn't pass beyond the mass. Definitive diagnosis required tissue biopsy. Endoscopic polypectomy can be attempted in patients with pedunculated polyps of less than $5 \mathrm{~cm}$ in size. However, in the setting of severe obstruction with polyps of $5 \mathrm{~cm}$ or larger, surgery is the preferred treatment. Similarly in our case, patient underwent distal gastrectomy with Billroth II reconstruction for symptomatic relief.

The presence of pancreatic tissue outside its normal localization and without anatomic and vascular continuity with the normal pancreas is termed pancreatic heterotopia (PH) [8]. The incidence reported is $1-2 \%$ as per autopsy studies. $\mathrm{PH}$ is known to be associated with various other associated anomalies like malrotation, small intestinal atresias, biliary atresias, congenital diaphragmatic hernias, Meckel's diverticuli, annular pancreas and esophageal atresias. It is most commonly found in the stomach $(25-38 \%)$, duodenum (17-21\%) and proximal jejunum (15-21\%), although it can occur anywhere in the gastrointestinal tract, pelvis, liver, biliary tract, spleen, omentum, mesentery, fallopian tube, mediastinum and lung $[9,10]$. It is usually asymptomatic and found during endoscopy or surgery, but may become clinically evident depending on the location (GOO in a prepyloric $\mathrm{PH}$ ). The symptoms are more likely in lesions larger than $2 \mathrm{~cm}$ [9]. The diagnosis of $\mathrm{PH}$ is difficult as there are no specific diagnostic methods. Though imaging studies can be helpful, definitive diagnosis is reached on histology and hence, surgery is frequently required to make a definitive diagnosis as in our case.

\section{Conclusion}

In summary, we presented a unique case of combination of three rarer diseases (EGE, BGH and $\mathrm{PH}$ ) manifesting together as GOO. Diagnosis of the eosinophilic gastroenteritis in the absence of obstruction may avoid surgical intervention and subsequent morbidity as medical treatment is often effective. However, in our case, endoscopic biopsies were inconclusive and the severity of symptoms required surgical intervention and diagnosis was made postoperatively. Cases of heterotopic pancreas and Brunner's gland hyperplasia causing GOO are very rare and similarly preoperative diagnosis is difficult. Therefore, they should be considered in the differential diagnosis of GOO. Though there is no special reason for the development of all the three lesions in one site in this case other than being coincidental, their presence together makes it a unique case.

\section{References}

[1] Appasani S, Kochhar R, Nagi B, Gupta V. Benign gastric outlet obstruction--spectrum and management. Trop Gastroenterol. 2011; 32(4):259-66.

[2] Mori A, Enweluzo C, Grier D, Badireddy M. Eosinophilic gastroenteritis: Review of a Rare and Treatable disease of the Gastrointestinal Tract. Case Rep Gastroenterol 2013; 7(2):293-8.

[3] Talley NJ, Shorter RG, Phillips SF, Zinmeister AR. Eosinophilic Gastroenteritis:a clinicopathological study of patients with disease of mucosa, muscle layer and subserosal tissues. Gut. 1990; 31:54-5.

[4] Chaudhary R, Shrivastava RK, Mukhopadhyay HG, Diwan RN, Das AK. Eosinophilic gastritis--an unusual cause of gastric outlet obstruction. Indian J Gastroenterol 2001; 20(3):110.

[5] Suraweera DB, Amin J, Baltayan A, Hu R. Brunner's Gland Hyperplasia: A Rare Cause Of Gastric Outlet Obstruction and a Review of Treatment Strategies. J Gastroenterol Hepatol Res. 2015; 4(7):1698-701. 
[6] Krishnamurthy P, Junaid O, Moezzi J, MD, Ali SA, Gopalswamy N. Gastric outlet obstruction caused by Brunner's gland hyperplasia: case report and review of literature. GIE.2006; 64(3):464-67.

[7] Dhinakar M, Allaya DJ, Golash. A Rare Case of Brunneroma Duodenum causing Gastric Outlet Obstruction. OMJ. 2010;25: 44-46.

[8] Deshpande VP, Raghunath BV, Sarin YK, Sinha S. Heterotopic pancreas causing duodenal obstruction in a patient previously treated for choledochal cyst. J Indian Assoc Pediatr Surg. 2012; 17(1):40-2.

[9] Trifan A, Tarcoveanu E, Danciu M, Hutanasu, Cojocariu C. Gastric Heterotopic Pancreas:An unusual case and Review of Literature. J Gastrointestin Liver Dis. 2012; 21(2):209-12.

[10] Ormarsson OT, Haugen SE, Juul I. Gastric outlet obstruction caused by Heterotopic pancreas. Eur J Pediatr Surg. 2003; $13(6): 410-3$. 